\title{
Analysis and Corresponding Measures to Factors about Effecting the High Availability of Digital Campus
}

\author{
Li Xinhua \\ Educational-Technology Center, \\ Bengbu Automobile Petty Officer Academy of PLA \\ Bengbu Anhui ,China \\ e-mail: lixinhua@ah.edu.cn
}

\begin{abstract}
The digital campus is a main platform for carrying out education information of colleges, its security and stability can directly affect the education, scientific research and management of colleges whether can normal proceed. This paper analyzes the four main factors about effecting the high availability of Digital Campus, its include Server Hosts, Storage, Network and Virus. In view of the four factors, we give the corresponding measures to improve the high availability of Digital Campus.
\end{abstract}

Keywords-Digital Campus;High Availability; Network Security

\section{INTRODUCTION}

Digitize campus is with the support of digitized information, using computer technology and network technology education to realize the teaching, scientific research and management, technical service and so on campus information collection, processing, integration, storage, transport, application, provide service platform and promote the development of the teaching and scientific research for the teaching and scientific research through the organization and business process reengineering, provide management platform for the school's management, improve the efficiency of the administrative management inside the school, provide open digital lives environment for the teachers and students[1]. Digital campus once built enabled, will become the school teaching, scientific research and management important information platform, and its robustness and reliability will affect the school daily the normal work of. This paper first analyzes the impact of digital campus high availability of the main factors, and then put forward some countermeasures for these factors.

\section{What IS A High AVAilabiLity?[2]}

High availability broadly refers to the system high availability. The calculation method of high availability in general with available per year to calculate level, such as regulations the year the whole system can reach 99.95\% environment, then the time of discontinuation maintenance system is $365 \times 24 \times(1-99.95 \%)=4.38$ hours. In addition, the availability of subsystem higher than the whole system availability, such as specified above the whole system availability is $99.95 \%$, for the subsystem, the availability is likely will reach $99.99 \%$. The available level and downtime comparison table of high availability are shown in Figure 1.

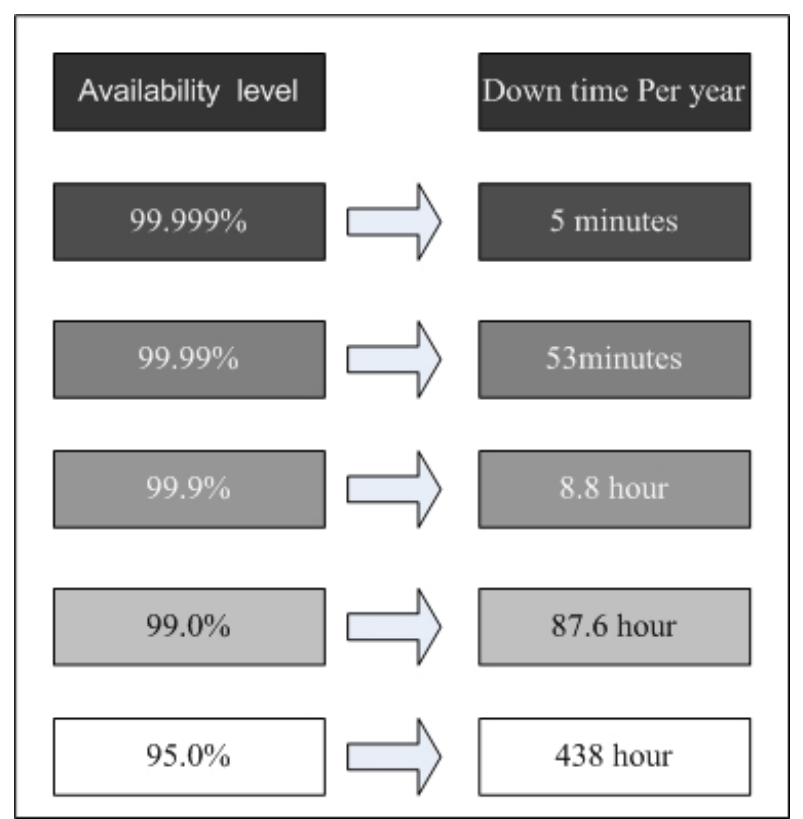

Figure 1. High availability level comparison table

No real $100 \%$ representation of the environment can be reached in real use environment. If it is $100 \%$ of online ability, we have to pay very big price. So usually reached more than $99.9 \%$ of the availability environment, generally it can be considered relatively high available environment.

\section{INFLUENCE FACTORS OF HIGH AVAILABILITY}

Digital campus construction is a large systematic project, including hardware facilities, network environment and system support software, etc. The whole system can be stable, healthy operation, to reach high availability goals, will be affected by a variety of factors, sum up mainly in the following aspects.

\section{A. Server Host}

A large server host running in digital campus system, used to support unified portal, identity authentication, and other various network application the operation of the system. These hosts, especially the key host, once appear fault, and will impact on the availability of digital campus. Host common fault including: operating system fault; Hardware failures; the power failure, etc. 


\section{B. Storage}

Digital campus is the information of collecting, sorting platform, a large amount of data to safe storage, such as personal information, authentication information, common card information and server information, etc. Storage problems result in significant data loss, can affect the whole system security and stability, and even cause the system can't run, which can reduce the system can be used level. The common failures of storage include: data jams, storage equipment failure, and etc.

\section{Network}

The network composed by the communication lines and node equipment, is the basis of digital campus operation, is the information circulation, interactive channel. The stability of the network to the reliable operation of the digital campus plays an important role, once a network in amongst the interrupt or key nodes equipment failure will be able to reduce the level of digital campus available. Common network fault including: line fault, exchange equipment failure, network congestion.

\section{Viruses, Trojan Horses and Malicious Attacks}

The rapid development of network technology, mobile storage media widely used, accelerates the viruses, Trojan horses to transmit between in the end user. The computer security of the end user related to the whole of the digital campus security level, the virus in the end user broke once, will take up lots of network bandwidth and further violations system service resources, so as to reduce the level of digital campus available. In addition, all kinds of hackers scanning, invasion, DDos attack threat digital campus software platform and all kinds of information system security. The operating system vulnerabilities, website and software design vulnerabilities for such an attack open the door. Once the successful attack, the important information in digital campus will face to be tampered and theft. In 2008, the attacker used of domestic famous university website two holes, through the SQL injection attack, tampering with the home page, the web server was crash, and caused a bad influence [3]. Viruses, Trojan horses and malicious attacks brought harm mainly include: the spread of the virus caused network congestion; the operating system is destroyed; system important data theft or tampered; attacks by the network congestion, etc.

\section{HIGH AVAILABILITY DESIGN OF DIGITAL CAMPUS}

\section{A. High Availability Design of Network}

- Rational planning of network. We should evaluate flow distribution for campus network, rational allocation network resources, in view of the large flow area, and provide a redundancy lines and equipment; We can realize load balance through ring network mode and setting strategy routing; We should rationally divided VLAN, reduce the network storm occurrence.

- Efficient network management software. High performance network management software system can monitor the router and switch, server and any other network node which support the SNMP, promptly discovery network fault, monitor network flow distribution, effective manage network availability and performance bandwidth, and even monitor to the server load, RAM usage, available disk space. As shown in figure 2 shows dynamic display of network monitoring software for Hefei University of Technology. Administrators can realtime monitor such flux distribution network, and solve the network congestion problem, enhance the digital campus usability level.

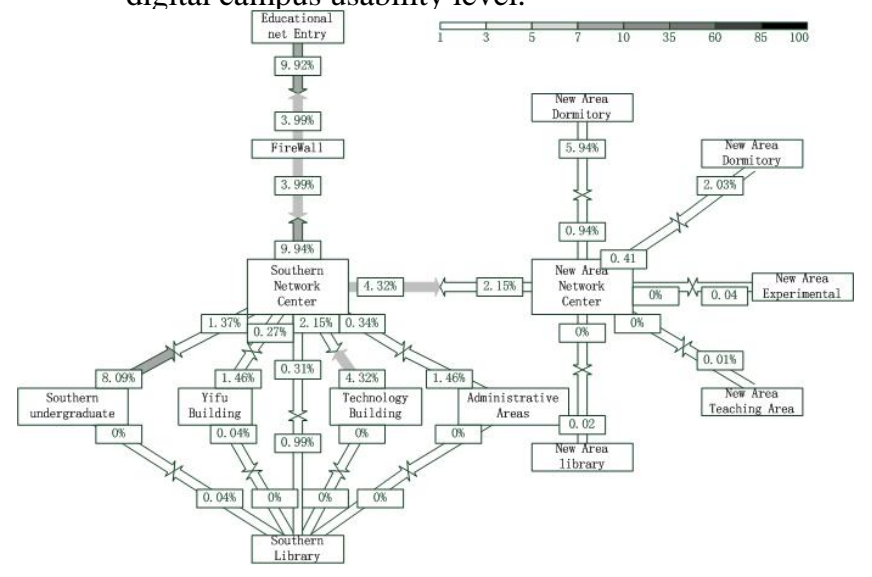

Figure 2. Dynamic display of network monitoring for Hefei University of Technology

\section{B. High Availability Design of Sever Host}

- The reasonable selection of host. For the host to provide service, we should fully consider the user capacity, use frequency, service resources consumption, select the appropriate host, avoid the server with overload appear when machine failure.

- Using cluster technology. We should use cluster technology protection for the hosts provided the key to important services, to improve the fault tolerance of host recovery capacity. In practical applications, we can choose the right cluster according to the circumstances. As shown in figure 3 shows the server cluster typical application. 


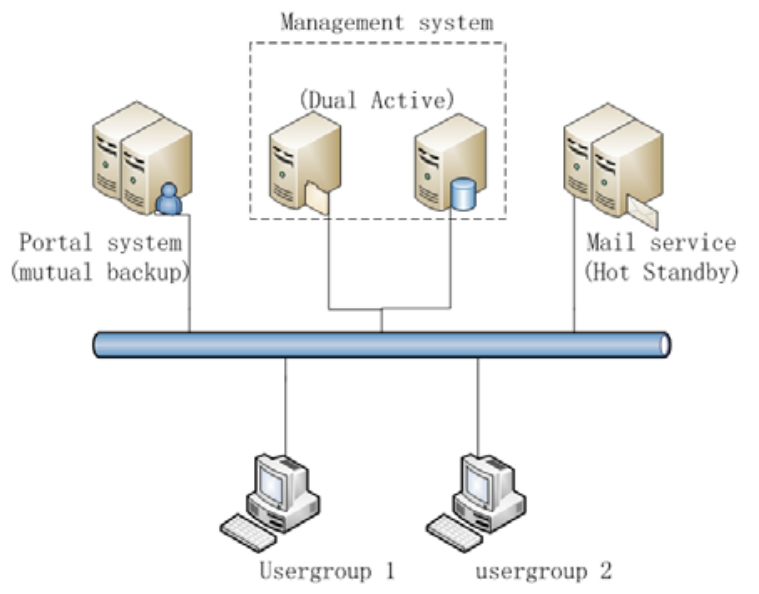

Figure 3. Server cluster typical application

\section{High Availability Design of Storage}

- Reasonable configuration storage devices. We should adopt appropriate storage equipment according to different application in digital campus, reasonable and effective use of resources. For important application service such as WWW/mail, card system and important data resources such as teaching resource center, digital library, can use the high speed, high bandwidth and high security FC SAN storage system. But for general application requirements such as entertainment, software download service can use cost-effective NAS system for storage.

- Using RAID technology. RAID is technologies which put more physical disk together in a certain way and form a huge logic disk, is a mature improves disk performance and redundancy of the technology. In the application of digital campus, according to the different application. We use reasonable RAID ways to configure the disk storage server, and can effectively improve the performance level of the application system available.

- Doing data backup work. Data backup is the foundation to improve the tolerant level of the system, can use tape library and recovery software combination to execute the centralized backup and recovery plan, realize high capacity, key task, zero down time, LAN-Free backup and recovery, and can satisfy the combination of large server centralized backup and recovery needs [4].

\section{Virus and Attack Prevention}

- Establishing safe protection system. Through the deployment of the firewall, intrusion detection system, prevent virus, gateway, and vulnerability scanning system, network audit system and other security protection equipment, establishing network boundary and internal protection system, can effectively reduce the attack and outbreaks, and improve the level of the digital campus available. As shown in figure 4 shows is the protection system structure:

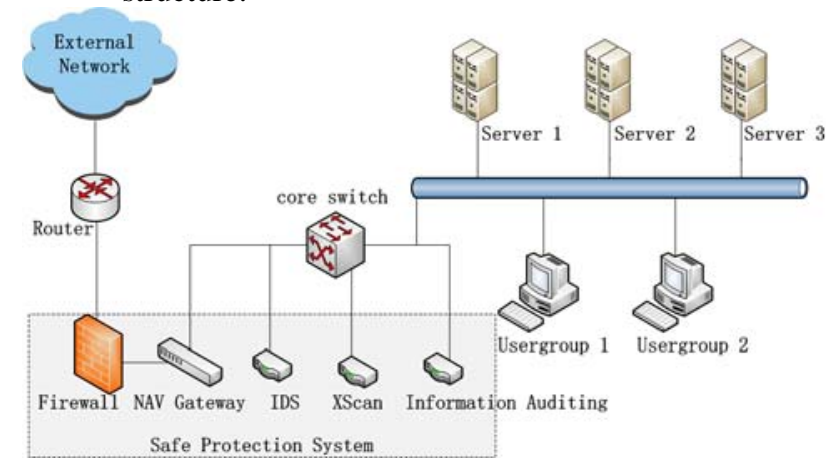

Figure 4. Typical safe protection system structure

- Providing network antivirus and automatically updating service for operating system. Through the deployment of online anti-virus software, may provide services of timing, forced antivirus, automatic distributing upgrade package to the digital campus within the system server, and end user, timely upgrades virus library users, and can also through the anti-virus software services within the program monitoring network virus outbreak, distribution, which convenient for administrator to take timely measures; Through the deployment of automatically update service to Windows operating system, timely repair the end user operation system vulnerability, and improve terminal computer of antiviral capacity.

- Improving security for application system code. Digital campus contains a number of network application system, in the deployment we should choose mature safe products, to provide good aftersales service, software vulnerabilities can timely repair. When developed application system by ourselves, we must pay special attention to against common code loophole attack, such as SQL injection, cross web scripting attack, etc, and always tracking software application situation, found that the problem in time of repair.

- Improving user protection level. We should periodically provide network security and virus protection knowledge training for the digital campus users, improve safety awareness and protection ability of users, from terminal to reduce virus and the behavior of the attack occurred.

\section{CONCLUSION}

With the rapid development of education information, the application of digital campus deepens, the dependence of the school teaching, scientific research and management system to the digital campus is growing. Security and stability of digital campus system are influenced by various factors, which need to seriously consider in digital campus construction and later the maintenance. We can through the 
reasonable planning and design, strict management monitoring and timely service guarantee these measures, to maximize digital campus availability level.

\section{REFERENCES}

[1] Jing YongWen, Zhang LiJing, and Xiong Wei, "Practice and Strategy of Digital Campus Construction,” China Electric Power Education, pp. 305-307, S4 2006.

[2] J. Clerk Maxwell, A Treatise on Electricity and Magnetism, 3rd ed., vol. 2. Oxford: Clarendon, 1892, pp.68-73.
[3] Zhang Hongjun, Zhang Xingang, “Analysis and Typical Security Measures for Digital Campus,” China Educational Technology, pp. 114115, June 2006.

[4] Shang Ercong, Zhang Yongqiang, "Research and Practice of Data Center Architecture," China Education Network, pp. 67-69, December 2007.

[5] Xue Ping-Chen, "SQL Injection Attack and Guard Technical Research,” CEIS 2011, Volume 15, 2011, Pages 4131-4135. 